ARTICLE

DOI: $10.1038 / s 41467-018-06352-5$

\title{
Uniform doping of graphene close to the Dirac point by polymer-assisted assembly of molecular dopants
}

Hans He ${ }^{1}$, Kyung Ho Kim¹,2, Andrey Danilov', Domenico Montemurro', Liyang Yu ${ }^{3}$, Yung Woo Park ${ }^{2,4,5}$, Floriana Lombardi (1) ${ }^{1}$, Thilo Bauch ${ }^{1}$, Kasper Moth-Poulsen (1) ${ }^{3}$, Tihomir lakimov ${ }^{6}$, Rositsa Yakimova ${ }^{6}$, Per Malmberg (i] ${ }^{3}$, Christian Müller (10 ${ }^{3}$, Sergey Kubatkin ${ }^{1} \&$ Samuel Lara-Avila (1) ${ }^{1,7}$

Tuning the charge carrier density of two-dimensional (2D) materials by incorporating dopants into the crystal lattice is a challenging task. An attractive alternative is the surface transfer doping by adsorption of molecules on 2D crystals, which can lead to ordered molecular arrays. However, such systems, demonstrated in ultra-high vacuum conditions (UHV), are often unstable in ambient conditions. Here we show that air-stable doping of epitaxial graphene on $\mathrm{SiC}$-achieved by spin-coating deposition of 2,3,5,6-tetrafluoro-tetracyano-quino-dimethane (F4TCNQ) incorporated in poly(methyl-methacrylate)-proceeds via the spontaneous accumulation of dopants at the graphene-polymer interface and by the formation of a charge-transfer complex that yields low-disorder, charge-neutral, large-area graphene with carrier mobilities $\sim 70000 \mathrm{~cm}^{2} \mathrm{~V}^{-1} \mathrm{~s}^{-1}$ at cryogenic temperatures. The assembly of dopants on 2D materials assisted by a polymer matrix, demonstrated by spincoating wafer-scale substrates in ambient conditions, opens up a scalable technological route toward expanding the functionality of $2 \mathrm{D}$ materials.

\footnotetext{
${ }^{1}$ Department of Microtechnology and Nanoscience, Chalmers University of Technology, 41296 Gothenburg, Sweden. ${ }^{2}$ Department of Physics and Astronomy, Seoul National University, Seoul 08826, Korea. ${ }^{3}$ Department of Chemistry and Chemical Engineering, Chalmers University of Technology, 41296 Göteborg, Sweden. ${ }^{4}$ Institute of Applied Physics, Seoul National University, Seoul 08826, Korea. ${ }^{5}$ Department of Physics and Astronomy, University of Pennsylvania, Philadelphia, PA 19104, USA. ${ }^{6}$ Department of Physics, Chemistry and Biology, Linkoping University, 58183 Linköping, Sweden. ${ }^{7}$ National Physical Laboratory, Hampton Road, Teddington TW11 OLW, UK. Correspondence and requests for materials should be addressed to S.L-A. (email: samuel.lara@chalmers.se)
} 
$\mathrm{H}$ omogeneous doping of graphene down to low carrier densities enables the study of delicate and fascinating physics at the Dirac point. The prime experimental archetypes for the study of Dirac physics in solid-state devices are van der Waals (VdW) hetero-structures of graphene and hexagonal boron nitride (hBN). In these, extremely low carrier densities in graphene are attainable, especially when prepared on atomically flat, electrically conductive substrates that screen longrange potential fluctuations (i.e. disorder) in the graphene layer ${ }^{1,2}$. Typically microscopic in size, VdW hetero-structures are prone to size-induced mesoscopic effects that obscure novel physical effects expected in graphene at charge neutrality. Largearea graphene, uniformly doped to the Dirac point as in the case of VdW hetero-structures, is required to unveil the intimate details of, for instance, metal-insulator transitions ${ }^{3}$, interacting Dirac fermions ${ }^{4}$, and Zitterbewegung of electrons in graphene ${ }^{5}$. Atomically flat, large-scale epitaxial graphene on $\mathrm{SiC}(\mathrm{SiC} / \mathrm{G})$ has demonstrated its potential for scalability in applications that demand extremely high-quality graphene over large areas, including quantum metrology 6,7 and high-frequency electronics 8,9 . Yet, in practice, carrier density control of $\mathrm{SiC} / \mathrm{G}$ remains a challenge because of high intrinsic n-doping $\left(n>10^{13}\right.$ $\mathrm{cm}^{-2}$ ) due to interaction with the substrate, making it difficult to substantially tune its carrier concentration ${ }^{10}$. While numerous gating techniques have been applied to $\mathrm{SiC} / \mathrm{G}$ to reduce its carrier density ${ }^{8,9}$, such approaches introduce carrier density fluctuations, which prevent further explorations of Dirac physics in this material.

Here we show a molecular approach to control the carrier density of epitaxial graphene homogeneously over macroscopic areas, yielding graphene with low carrier density $\left(n<10^{10} \mathrm{~cm}^{-2}\right)$, low charge fluctuations (at the level of $\Delta n \approx \pm 6 \times 10^{9} \mathrm{~cm}^{-2}$ ), and carrier mobilities up to $70000 \mathrm{~cm}^{2} \mathrm{~V}^{-1} \mathrm{~s}^{-1}$ at low temperatures. So far, such electron transport properties have only been attained in microscopic single-crystal graphene flakes encapsulated by $\mathrm{hBN}^{11}$ or in suspended graphene $\mathrm{e}^{12}$. The doping method described here was applied to samples over $5 \times 5 \mathrm{~mm}^{2}$, resulting in epitaxial graphene devices that display quantum Hall effect at magnetic fields $B<1 \mathrm{~T}$. These measurements indicate, as we elaborate below, a highly homogeneous spatial distribution of charge carriers on graphene. Chemical analysis reveals that air-stable molecular doping of graphene is achieved when organic molecules, embedded in a polymer matrix, diffuse through the matrix and spontaneously accumulate at the graphene surface due to formation of a charge-transfer complex. High-electron-affinity 2,3,5,6-tetrafluoro-tetracyano-quino-dimethane (F4TCNQ) molecules are mixed in a liquid solution with poly(methyl-methacrylate) (PMMA) and spin-coated at ambient conditions on $\mathrm{SiC} / \mathrm{G}$, with graphene being used both as the target substrate for molecular assembly and, simultaneously, as a charge sensor. The stability of the samples allows us to study the chemical composition as well as electronic transport properties of the F4TCNQ/graphene system. We expect this method to open up a scalable route toward expanding the properties and functionality of graphene and other two-dimensional (2D) materials well beyond doping control.

\section{Results}

Doping schemes. The different chemical doping schemes of graphene devices are shown in Fig. 1b. Our devices include large $(W=5 \mathrm{~mm} \times L=5 \mathrm{~mm})$ (Fig. 1a) and small ( $W=2-100 \mu \mathrm{m} \times$ $L=10-180 \mu \mathrm{m})$ epitaxial graphene Hall bars fabricated by conventional electron beam lithography (see Methods section). When PMMA is used as a spacer between graphene and the molecular dopant layer, the carrier density decreases three orders of magnitude from its pristine value, $n \approx 1 \times 10^{13} \mathrm{~cm}^{-2}$ to near charge neutrality, $n \approx 1 \times 10^{10} \mathrm{~cm}^{-2}$ (Fig. 1c). Importantly, even at such low carrier densities the carrier mobility remains high, with the largest measured value exceeding $\mu \approx 50000 \mathrm{~cm}^{2}$ $\mathrm{V}^{-1} \mathrm{~s}^{-1}$ at $T=2 \mathrm{~K}$. The effect of doping is homogeneous over millimeter scale and large samples display quantum Hall effect at magnetic fields $B<1 \mathrm{~T}$ (Fig. 1a), retaining their low carrier density over the course of 2 years, even under ambient conditions (Supplementary Fig. 1). To achieve this, a 200-nm-thick layer of the PMMA-F4TCNQ dopant blend is spin-coated onto a PMMAprotected sample, followed by thermal annealing above the PMMA glass transition temperature. The resulting carrier density could be fine-tuned by the total annealing time. For a concentration of 7\% of F4TCNQ in PMMA by weight, charge-neutral graphene is achieved after annealing at $T=160^{\circ} \mathrm{C}$ for $5 \mathrm{~min}$. Shorter annealing times yield hole-doping and longer times yield electron-doping (Supplementary Fig. 1). Using the optimal time, we have consistently observed a decrease in electron density by three orders of magnitude together with a tenfold increase of carrier mobility at $T=2 \mathrm{~K}$ in more than 20 devices on 11 different chips (Supplementary Table 1 and Supplementary Fig. 2). Typical carrier concentrations in doped samples are of the order of $5 \times 10^{11} \mathrm{~cm}^{-2}$ at room temperature. They decrease to values $\sim 1 \times 10^{10} \mathrm{~cm}^{-2}$ at $T=2 \mathrm{~K}$, due to freezing of thermally activated carriers ${ }^{13}$. The corresponding carrier mobilities are in the range of $30000-50000 \mathrm{~cm}^{2} \mathrm{~V}^{-1} \mathrm{~s}^{-1}$ (Fig. 1d). The PMMA spacer layer plays a crucial role in achieving high carrier mobilities. While both PMMA and the dopant blend act independently as moderate p-dopants when deposited directly on $\mathrm{SiC} / \mathrm{G}$, it is only when the PMMA spacer layer is added between graphene and the dopant blend that we observe the near to charge neutrality doping effect. Similarly, carrier mobilities exceed $10000 \mathrm{~cm}^{2} \mathrm{~V}^{-1} \mathrm{~s}^{-1}$ only if the PMMA spacer and dopant blend operate in tandem (Supplementary Fig. 3).

Chemical analysis of graphene-polymer hetero-structure. We demonstrate that the doping of graphene is the result of F4TCNQ molecules diffusing through the PMMA layer and accumulating at the graphene surface. Figure $2 \mathrm{a}, \mathrm{b}$ shows the chemical depth profile of the polymer stack, obtained by using time-of-flight secondary ion mass spectrometry (ToF-SIMS), revealing both diffusion of F4TCNQ through the PMMA spacer and the accumulation of molecules at the graphene surface. From this we estimate the diffusion coefficient of F4TCNQ through PMMA to be of the order of $10^{14} \mathrm{~cm}^{2} \mathrm{~s}^{-1}$ and by integrating the areas under the ion current intensity curves, we estimate the density of F4TCNQ molecules near the graphene surface to be $\sim 4.6 \times 10^{14} \mathrm{~cm}^{-2}$ (Supplementary Fig. 4, Supplementary Note 1). Graphene and metallic surfaces promote the accumulation of F4TCNQ, while there are virtually no dopant molecules at the polymer/SiC interface. The surface density of F4TCNQ is roughly $50 \%$ greater on graphene (and sixfold higher on gold) compared to that in the dopant blend layer (Fig. 2c). We attribute the accumulation of F4TCNQ on the graphene surface and the measured p-doping effect to the formation of a charge transfer complex, with partially charged F4TCNQ remaining at the graphene interface to preserve overall charge neutrality. F4TCNQ is known to be mobile in thin polymer films ${ }^{14,15}$, with its diffusion depending on polarity and glass transition temperature of the polymer. When using an inert PMMA as a host matrix, F4TCNQ remains neutral both in the doping layer and as it diffuses through PMMA spacer layer ${ }^{16}$. The formation of a charge transfer complex takes place only when encountering an electron donor, such as graphene. Once charged, the F4TCNQ anion is bound to graphene, stabilized by Coulomb interaction ${ }^{17}$. 
a
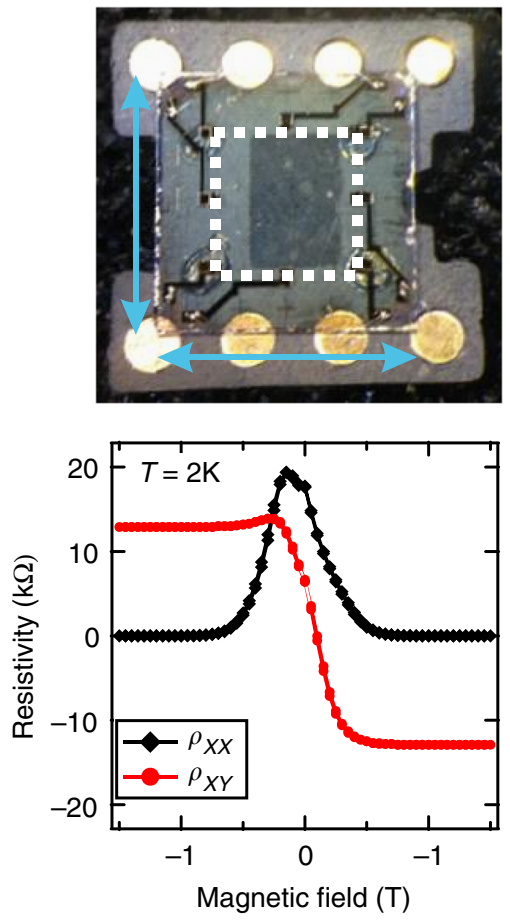

b
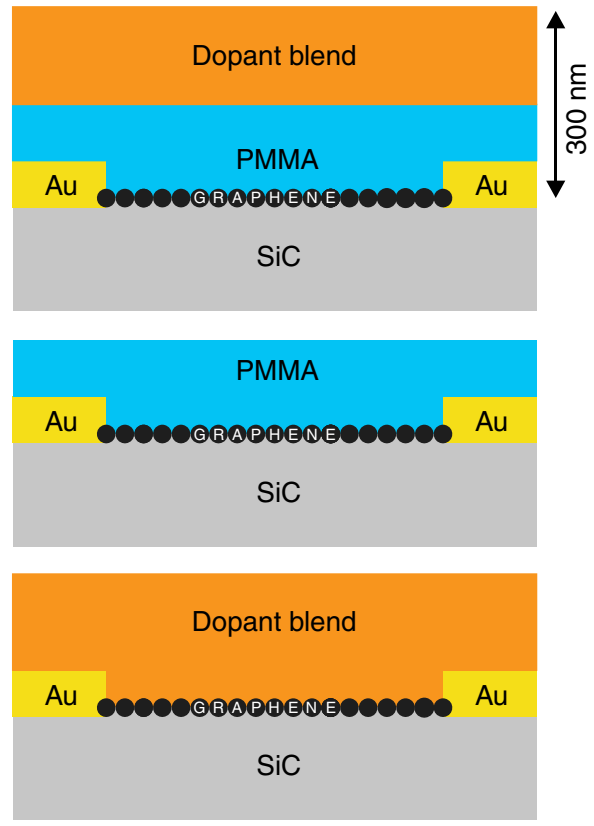

C
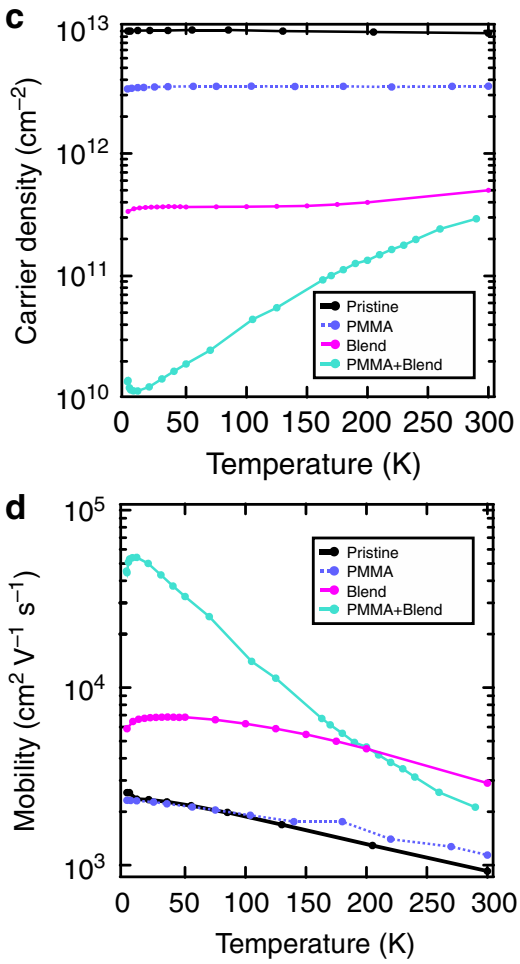

Fig. 1 Magnetotransport characterization of chemically doped SiC/G. a Top: macroscopic graphene Hall bar device (white dotted outline, $W=5 \mathrm{~mm} \times L=$ $5 \mathrm{~mm}$ ). Bottom: low field magnetoresistance and fully developed quantum Hall effect indicating low charge disorder in chemically doped graphene, even over macroscopic areas. For this device, the measured carrier density $p=9 \times 10^{9} \mathrm{~cm}^{-2}$ and mobility $\mu=67000 \mathrm{~cm}^{2} \mathrm{~V}^{-1} \mathrm{~s}^{-1} . \mathbf{b}$ The different encapsulation schemes with a polymer stack consisting of PMMA-F4TCNQ dopant blend, which comprises of F4TCNQ molecules in a PMMA matrix (F4TCNQ 7 wt\%). The three schemes are dopant blend separated from graphene by a PMMA spacer (top), only PMMA layer (middle), and the dopant blend directly on the surface of graphene (bottom). c Carrier density as a function of temperature extracted from Hall measurements on small epitaxial graphene devices ( $W=$ 2-50 $\mu \mathrm{m} \times L=4-180 \mu \mathrm{m})$. d The corresponding Hall carrier mobility showing the highest $\mu \sim 55000 \mathrm{~cm}^{2} \mathrm{~V}^{-1} \mathrm{~s}^{-1}$ at $T=10 \mathrm{~K}$ for sample prepared with PMMA spacer and dopant layer. The downturn in mobility at lower temperatures is due to quantum corrections to the Drude resistance. Carrier concentration $n$ and mobilities $\mu$ were extracted from Hall measurements as $n=1 / e R_{H}$ and $\mu=R_{H} / \rho_{X X}$, with e the elementary charge, the Hall coefficient $R_{\mathrm{H}}=\mathrm{d} R_{X Y} / \mathrm{d} B$, the longitudinal sheet resistance $\rho_{X X}=R_{X X} W / L$, and $R_{X Y}$ the transversal resistance

a

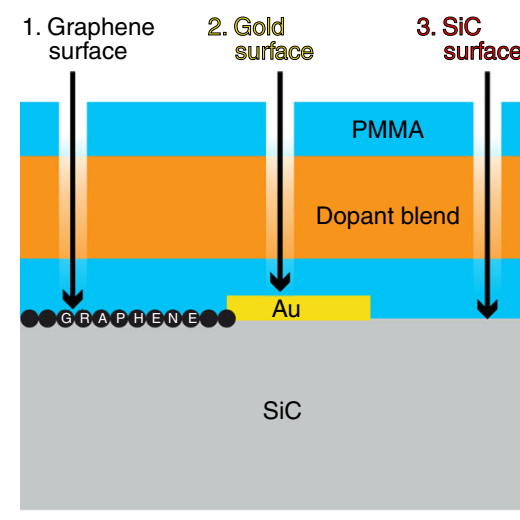

b
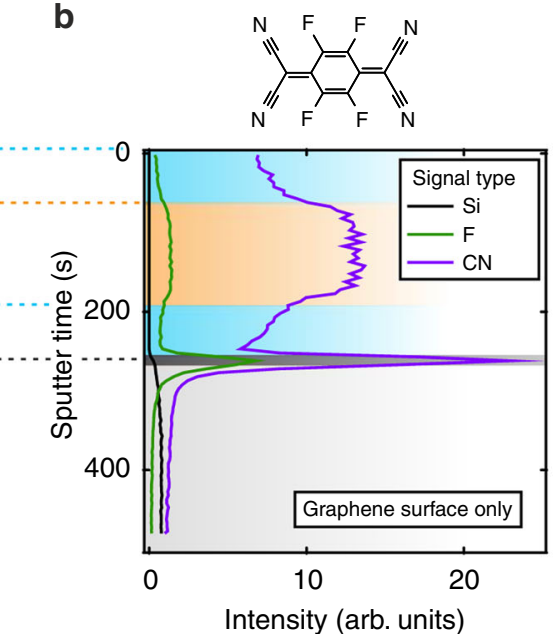

C

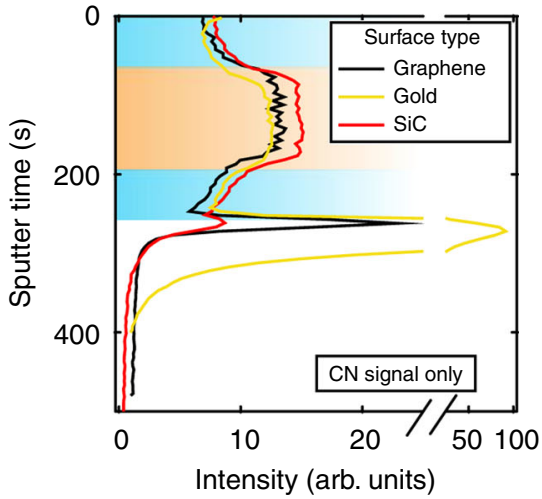

Fig. 2 Chemical profiling of the polymer layers using ToF-SIMS to detect fingerprints of the F4TCNQ molecule (F and CN ions) as one probes deeper into the polymer stack. a Samples were prepared with PMMA spacer, dopant blend, and PMMA encapsulation layer. Three distinct spots on the substrate have been investigated: (1) graphene, (2) gold, and (3) SiC surfaces. b When analyzing the polymer layers on spot 1, above the graphene surface, the ion intensity for $\mathrm{F}$ and $\mathrm{CN}$ ions (top inset shows a schematic representation of a F4TCNQ molecule) vs. sputter time reveals significant accumulation of F4TCNQ at the graphene/PMMA interface, as well as the spatial distribution of F4TCNQ molecules through the spacer and encapsulation PMMA layers. The onset of the silicon signal $(\mathrm{Si})$ is the marker, which indicates that the $\mathrm{SiC}$ substrate has been reached. The shaded regions denote the extent of each layer, from top PMMA layer down to SiC substrate. c Here we focus only on the $\mathrm{CN}$ signal measured at all three different spots. This analysis shows accumulation of F4TCNQ on the conductive surfaces of graphene and gold, but virtually no accumulation on SiC 
a

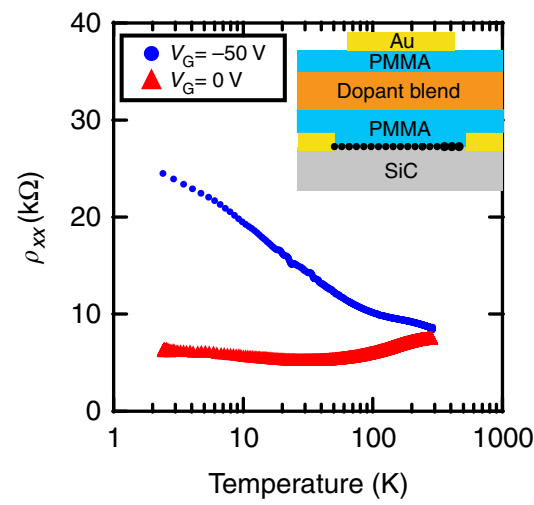

b

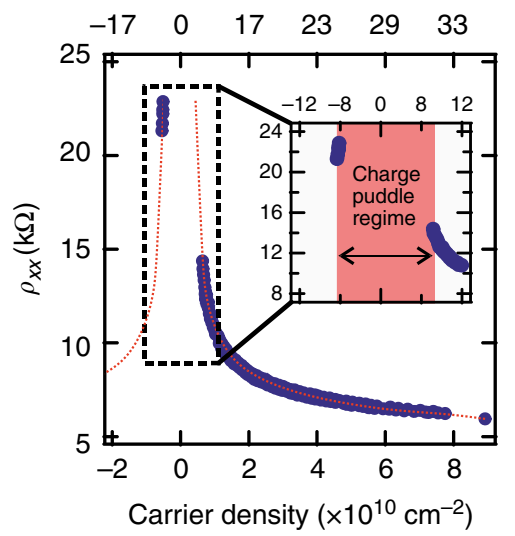

C

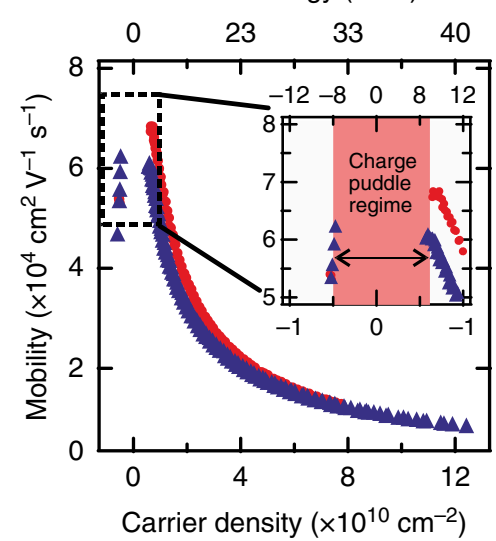

Fig. 3 Electrostatic gating of chemically doped graphene $(30 \mu \mathrm{m} \times 180 \mu \mathrm{m}$ Hall bar $)$. a Temperature dependence of longitudinal resistance for graphene in the metallic limit (red) and gated to Dirac point (blue). (Inset) Schematic representation of a chemically doped graphene device with a metallic gate (the topmost Au layer). $\mathbf{b} \rho_{X X}$ vs. carrier concentration shows the characteristic Dirac peak around the charge neutrality point (red dotted line serves as a guide to the eye). The Dirac point is crossed below $V g=-40 \mathrm{~V}$, and the device has well-defined carrier densities within $\pm 6 \times 10^{9} \mathrm{~cm}^{-2}$ at $T=2 \mathrm{~K}$, which corresponds to a Fermi energy of $\pm 9 \mathrm{meV}$. c Corresponding charge carrier mobilities, with values up to $70000 \mathrm{~cm}^{2} \mathrm{~V}^{-1} \mathrm{~s}^{-1}$. Each point in $\mathbf{b}$ and c represents data of magnetic field scans where $R_{X Y}$ is linear in the low magnetic field limit and the device shows fully developed quantum Hall effect at high magnetic fields ( $\rho_{X X}=0$ and exactly quantized $R_{X Y}$ plateaus). The gap in data around zero carrier concentration corresponds to omitted data points where graphene is in the charge puddle regime. For comparison, in c, red circles and blue triangles correspond to Hall measurements from two different Hall probe pairs on the same device. The gate voltage was applied at cryogenic temperatures; the measured leakage current did not exceed $50 \mathrm{pA}$, with the bias current on graphene of $100 \mathrm{nA}$

Low charge disorder in SiC/G doped close to Dirac point. We investigated further electron transport details of the F4TCNQgraphene charge-transfer complex system by introducing a top electrostatic gate (Fig. 3a, inset) on top of a $30 \mu \mathrm{m} \times 180 \mu \mathrm{m}$ Hall bar. The top gate enables additional fine tuning of the carrier concentration within $\Delta n \sim 5 \times 10^{11} \mathrm{~cm}^{-2}$ using gate voltages $V_{\mathrm{G}}=-100$ to $+200 \mathrm{~V}$. At $V_{\mathrm{G}}=0 \mathrm{~V}$ the carrier density is $n=5 \times$ $10^{11} \mathrm{~cm}^{-2}$ at room temperature and graphene is in the metallic limit. In this case, $\rho_{X X}\left(T, V_{\mathrm{G}}=0 \mathrm{~V}\right)$ decreases linearly with temperature from its room temperature value, due to suppression of acoustic phonon scattering. Quantum corrections to resistance result in a $\log (T)$ dependence below the Bloch-Grüneisen temperature $\quad T_{\mathrm{BG}}=2 v_{\mathrm{ph}} E_{\mathrm{F}} /\left(k_{\mathrm{B}} v_{\mathrm{F}}\right)=2 v_{\mathrm{ph}} \hbar v_{\mathrm{F}} \sqrt{\pi n} /\left(k_{\mathrm{B}} v_{\mathrm{F}}\right) \approx 38 \mathrm{~K}$, with the phonon velocity $v_{\mathrm{ph}}=2 \times 10^{4} \mathrm{~m} \mathrm{~s}^{-1}\left(E_{\mathrm{F}}=\hbar v_{\mathrm{F}} \sqrt{\pi n}\right.$ the Fermi level of graphene, $\hbar$ the reduced Planck's constant, $k_{\mathrm{B}}$ the Boltzmann constant, and $v_{\mathrm{F}}=10^{6} \mathrm{~m} \mathrm{~s}^{-1}$ the Fermi velocity in graphen $\left.{ }^{18}\right)$. In contrast, when graphene is gated to the Dirac point, the sheet resistance $\rho_{X X}(T, V g=-50 \mathrm{~V})$ monotonically increases as the temperature drops, though remains finite with no indication of a transport gap in the current voltage characteristics down to $T=2 \mathrm{~K}$ (Fig. 3a).

To characterize the magnitude of carrier density fluctuations (i.e. how precisely one can approach the Dirac point) we conducted low-temperature magnetotransport measurements on chemically and electrostatically gated devices and found these fluctuations to be on the level of $\Delta n \sim \pm 6 \times 10^{9} \mathrm{~cm}^{-2}\left(\Delta E_{\mathrm{F}} \sim \pm 9\right.$ $\mathrm{meV}$ ). Figure $3 \mathrm{~b}$ shows longitudinal resistance vs. carrier concentration in which every data point, extracted from individual measurements of $\rho_{X X}(B), R_{X Y}(B)$ at a fixed gate voltage, corresponds to devices behaving as a system with a single electronic band and spatially homogenous carrier density ${ }^{19-23}$. That is, data points in Fig. $3 \mathrm{~b}$ fulfill simultaneously the criteria of linear $R_{X Y}(B)$ at low fields ${ }^{19-22}$, and fully developed half-integer quantum Hall Effect at high fields ${ }^{23}$, i.e. $\rho_{X X}(B)=0 \Omega$ and strictly quantized $R_{X Y}$ plateau over the entire available range of magnetic field (Supplementary Fig. 5). The gap in data around zero carrier concentration thus corresponds to data points where graphene is in the charge puddle regime. Under quantizing conditions, the residual disorder in the sample causes non-zero and oscillatory $\rho_{X X}(B)$ once the magnetic length approaches the average charge puddle size ${ }^{23,24}$. In our samples with low carrier density concentration $\sim 10^{10} \mathrm{~cm}^{-2}$ we have observed no deviation from $\rho_{X X}(B)=0$ to the largest magnetic field available in our setup $(B=14 \mathrm{~T})$ (Supplementary Fig. 5). Thus, we establish an upper limit for the puddle size of about $l_{\mathrm{B}}=\sqrt{\hbar / e B} \approx 7 \mathrm{~nm}$. The charge puddle magnitude is directly connected to disorder introduced by, e.g., topography or inhomogeneous doping 25 . The small magnitude of charge puddles measured in our devices indicate that $\mathrm{SiC} / \mathrm{G}$ doped with F4TCNQ molecules is homogenous also at the microscopic scale, with carrier density fluctuations comparable to those in high-quality, hBNencapsulated single-crystal graphene flakes obtained by exfoliation $^{11}$ or by chemical vapor deposition growth ${ }^{26}$ (Supplementary Fig. 6).

It is remarkable that epitaxial graphene displays such low disorder even at extreme dopant coverage, being decorated with a dense layer of molecules of about 3-4 molecules per $\mathrm{nm}^{2}(c \approx 3 \times$ $10^{14} \mathrm{~cm}^{-2}$, comparable to the molecular coverage of $c \approx 4.6 \times$ $10^{14} \mathrm{~cm}^{-2}$ from SIMS). We estimated the molecular density at the graphene surface from the shift in carrier density measured in doped graphene with respect to its pristine concentration $(\Delta n \approx$ $1 \times 10^{13} \mathrm{~cm}^{-2}$ ), and assuming that 0.3 electrons are withdrawn from epitaxial graphene per molecule ${ }^{27,28}$ with $1 / 10$ gate efficiency ${ }^{10}$. The homogeneity in doping is in part enabled by the high degree of F4TCNQ dispersion inside the PMMA matrix, shown by room temperature grazing-incidence wide-angle X-ray scattering (GIWAXs). The diffractogram in Fig. $4 \mathrm{a}, \mathrm{b}$ reveals a broad amorphous halo with a distinct diffraction peak at $q=9.6$ $\mathrm{nm}^{-1}$ from PMMA (Supplementary Note 2). The absence of diffraction spots from F4TCNQ implies the lack of molecular aggregation (i.e. crystallites) inside the matrix. Given the size of the F4TCNQ molecule, we propose that at this packing density a 


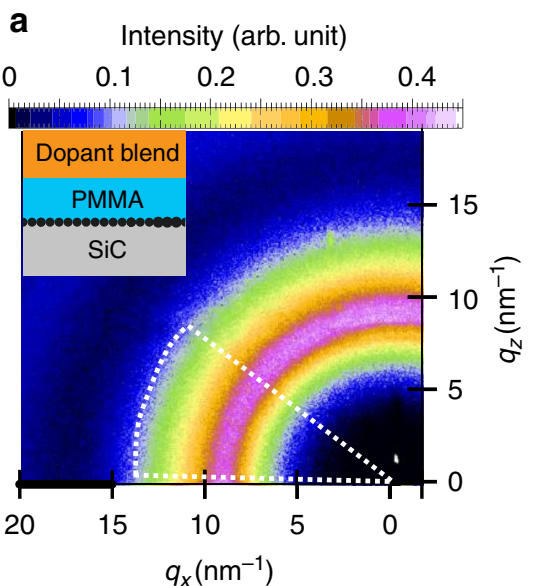

b

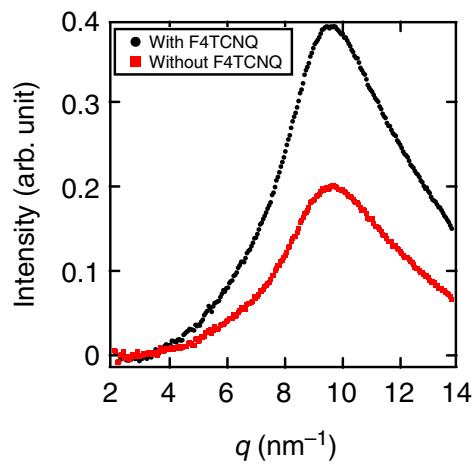

c

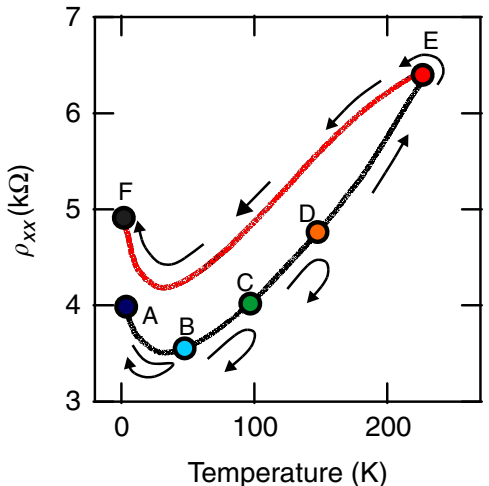

Fig. 4 F4TCNQ in PMMA matrix. a Grazing-incidence wide-angle x-ray scattering (GIWAXs) measurements taken on $\mathrm{SiC} / \mathrm{G}$ with dopant blend and PMMA spacer (inset) (ambient condition, room temperature, $0.15^{\circ}$ incident angle). The diffraction ring with radius $q=9.6 \mathrm{~nm}^{-1}$ from PMMA and the absence of F4TCNQ diffraction spots suggests a good molecular solubility in the polymer matrix. The color bar shows the normalized intensity, after a constant diffuse scattering background has been subtracted. The white dotted region denoted over which azimuthal angles the intensity profile in $\mathbf{b}$ has been averaged. $\mathbf{b}$ The reference sample, without F4TCNQ in the dopant blend displays a clear peak diffraction ring with radius $q=9.6 \mathrm{~nm}{ }^{-1}$; the addition of F4TCNQ molecules enhances the signal twofold. c Stability of doping at low temperature in a device that has been cooled down from room temperature. Starting at $T=300 \mathrm{~K}$ we applied a gate voltage $V_{\mathrm{G}}=+50 \mathrm{~V}$ during cool down. Once we reached $T=2 \mathrm{~K}$, the gate terminal is set to $V_{\mathrm{G}}=0 \mathrm{~V}$ and sample sheet resistance acquires a value of $\rho_{X X}=4 \mathrm{k} \Omega$ (point A). Thermal excursions to $T=50 \mathrm{~K}(\mathrm{~B}), 100 \mathrm{~K}\left(\mathrm{C}\right.$ ), and $150 \mathrm{~K}(\mathrm{D})$ result in reversible $\rho_{X X}(T)$ along the black curve. Once temperature exceeds $T=150 \mathrm{~K}(\mathrm{E}), \rho_{X X}(T)$ irreversibly changes to a higher resistive value (red curve), and on cooling down back to $T=2 \mathrm{~K}$, the sample resistance takes a value of $\rho_{X X}=5 \mathrm{k} \Omega(\mathrm{F})$. In the absence of the gate voltage, the sample resistance remains in the higher resistance $\rho_{X X}(T)$ branch

feasible molecular orientation of F4TCNQ is close to that of molecules standing up on the graphene surface ${ }^{28}$. Yet, we do not rule out molecular re-orientation or thermally induced redistribution of charges in the dopant layer under the effect of electric field, even at low temperatures. Such charge redistribution in the dopants in close vicinity to graphene may be responsible for screening charge inhomogeneities that facilitate highly uniform doping ${ }^{1}$. Thermally activated motion of charges in the dopant layer is a plausible source of the hysteresis in $\rho_{X X}(T)$ when devices are subjected to thermal excursion from $T=2$ to $230 \mathrm{~K}$ (Fig. 4c). This is also suggested by more accurate resistance measurements, which reveal that charges in the dopant layer are mobile down to $T \approx 113 \mathrm{~K}$ (Supplementary Fig. 7), in notable coincidence with the energy scale of the measured charge inhomogeneity in doped epitaxial graphene $\left(\Delta E_{\mathrm{F}} \sim \pm 9 \mathrm{meV}\right)$. The charge homogeneity of the samples is thus determined by the temperature at which the screening charges freeze.

\section{Discussion}

A possible explanation for the observed low disorder and high charge carrier mobility of $\mathrm{SiC} / \mathrm{G}$ at large molecular coverage is a high degree of spatial correlation between adsorbed F4TCNQ and impurities present on bare graphene. Together with the low charge carrier density fluctuations, increasing impurity (e.g. F4TCNQ) density can lead to suppression of charge scattering in graphene if there is sufficient inter-impurity correlation present in the system ${ }^{29,30}$ (Supplementary Fig. 8). The actual organization and conformation of charged molecules on graphene is the result of delicate balance between molecule-graphene interactions as well as the intermolecular many-body dispersion forces ${ }^{31}$ in the presence of polymer. While the exact arrangement of molecules on graphene is difficult to probe through the thick polymer layers with surface science techniques, the graphene layer itself utilized as detector allows gaining an insight of such molecular reorganization at the polymer-graphene interface.
In summary, we presented a method of guiding the assembly of molecular dopants onto the surface of graphene by using an organic polymer matrix. With the doping stability observed in our samples, the tuneability of molecular coverage, and given the vast catalog of polymers and organic/organo-metallic molecules, we expect this method to open up a scalable route toward expanding the properties and functionality of graphene and other 2D materials well beyond doping control. Moreover, the presented analysis of chemical and electron transport properties of doped graphene sheds light on the complex processes that molecular dopants undergo when embedded in polymers. This is relevant to the understanding of performance, materials, and interfaces in organic electronic devices, especially when combined with $2 \mathrm{D}$ materials. This method can be explored in the future to create and study electron transport properties of novel $2 \mathrm{D}$ systems of ordered molecular arrays templated by $2 \mathrm{D}$ crystals $^{32-35}$.

\section{Methods}

Sample fabrication. Monolayer $\mathrm{SiC} / \mathrm{G}$ was grown on the $\mathrm{Si}$-face of $\mathrm{SiC}$ using thermal decomposition of $7 \mathrm{~mm} \times 7 \mathrm{~mm} 4 \mathrm{H}(0001)$-SiC substrates. The samples were grown in argon atmosphere of $800 \mathrm{mbar}$, and kept at a temperature of around $1700{ }^{\circ} \mathrm{C}$ for $5 \mathrm{~min}^{36}$. Optical microscopy ${ }^{37}$ revealed a typical surface coverage of $>95 \%$ monolayer graphene.

Epitaxial graphene Hall bars were fabricated using electron beam lithography described in detail elsewhere ${ }^{38}$. The electrical contacts were fabricated using physical vapor deposition of $\mathrm{Ti}$ and $\mathrm{Au}, 5$ and $80 \mathrm{~nm}$ thick, respectively. After fabrication samples were cleaned using isopropyl alcohol, acetone, and dried using nitrogen gas. For pristine $\mathrm{SiC} / \mathrm{G}$ (shown in Fig. 1b) a hBN flake was exfoliated and transferred to pristine $\mathrm{SiC} / \mathrm{G}$ using a polydimethylsiloxane stamp. To clean the interface the sample was annealed in argon 800 mbar at $755^{\circ} \mathrm{C}$, after which standard lithography was performed.

The dopant blend consisted of a mixture of F4TCNQ (Sigma-Aldrich) and PMMA (Microlithography Chemicals Corp., molecular weight 950000 ). A unit of $25 \mathrm{mg}$ of dry F4TCNQ powder was mixed with $3 \mathrm{ml}$ anisole solvent. A volume of $0.5 \mathrm{ml}$ of this solution was then mixed with $1 \mathrm{ml}$ PMMA A6 (6\% PMMA by weight in anisole). The PMMA spacer layer and encapsulation layers used either PMMA A4 (5\% by weight in anisole) or PMMA-based copolymer (MMA (8.5) MAA) EL6 (6\% by weight in ethyl lactate solvent). All polymer layers were deposited on graphene using spin-coating at $6000 \mathrm{rpm}$ for $1 \mathrm{~min}$. After each spin-coating step a 
5 min baking step on a hotplate set at $160^{\circ} \mathrm{C}$ follows. The dopant blend is an exception, since the baking time dictates the final doping level.

The electrostatic top gate was fabricated using a shadow mask, and physical vapor deposition of $\mathrm{Au}$ in order to avoid disruption of the chemically doped sample. In order to preserve the integrity of the doping strength of graphene, additional layers of dopant blend and PMMA encapsulation layers were deposited before fabrication of the gate. The samples with the full stack of five polymer layers are the most resilient to drift in carrier concentrations in ambient conditions.

Electrical measurements. Standard electrical characterization was performed in a liquid ${ }^{4} \mathrm{He}$ gas flow cryostat, which allowed for temperatures down to $2 \mathrm{~K}$ and magnetic fields up to $14 \mathrm{~T}$. All reported values of charge carrier mobility and charge carrier concentration were extracted from four-probe Hall and quantum Hall measurements. The standard measurement setup used current biased samples at maximum of $100 \mathrm{nA}$ DC (Keithley $6221 \mathrm{DC}$ and AC current source, Agilent 34420 A nanovolt meter), in this range samples showed linear $I V$ characteristics down to cryogenic temperatures. The electrostatic top gate was DC biased with voltages ranging from -100 to $+200 \mathrm{~V}$, where leakage current was $<0.05 \mathrm{nA}$.

Secondary ion mass spectrometry. ToF-SIMS analysis was performed using a TOF.SIMS 5 instrument (ION-TOF GmbH, Münster, Germany). The instrument is equipped with a $25 \mathrm{keV} \mathrm{Bi}_{3}{ }^{+}$cluster ion gun as the primary ion source and a 10 $\mathrm{keV} \mathrm{C}_{60}+$ ion source for sputtering and etching. The samples were analyzed using a pulsed primary ion beam $\left(\mathrm{Bi}_{3}{ }^{++}, 0.34 \mathrm{pA}\right.$ at $\left.50 \mathrm{keV}\right)$ with a focus of approximately $2 \mu \mathrm{m}$ at a field of view of $150 \mu \mathrm{m} \times 150 \mu \mathrm{m}$. The mass resolution using this setup was at least $M / \Delta M=5000$ full width at half maximum at $\mathrm{m} / z 500$. All spectra were acquired and processed with the Surface Lab software (version 6.4, ION-TOF $\mathrm{GmbH}$ ). Depth profile analysis was performed using a $\mathrm{C}_{60}{ }^{++}$beam at $20 \mathrm{keV}$ with a current of $0.2 \mathrm{nA}$ in a non-interlaced mode with $1 \mathrm{~s}$ of analysis, $1 \mathrm{~s}$ of sputtering, and a pause of $1 \mathrm{~s}$ for each sputter cycle at $350 \mu \mathrm{m} \times 350 \mu \mathrm{m}$. The maximum ion dose density of $\mathrm{Bi}_{3}{ }^{++}$was kept between $4 \times 10^{12}$ and $7 \times 10^{12} \mathrm{~cm}^{-2}$ over the whole depth profiling experiment, while the ion dose for $\mathrm{C}_{60}{ }^{++}$ranged from $2 \times 10^{14}$ to $4 \times 10^{14}$ ions per $\mathrm{cm}^{2}$. Low-energy electrons were used for charge compensation during analysis.

Grazing-incidence wide-angle X-ray scattering. Data were obtained at the D1beam line at the Cornell High Energy Synchrotron Source at Cornell University. The measurements used a positron beam with synchrotron radiation of a wavelength of $1.162 \AA$, Pilatus $200 \mathrm{~K}$ detector with pixel size of $172 \mu \mathrm{m} \times 172 \mu \mathrm{m}$ and sample to detector distance of $177.2 \mathrm{~mm}$. Measurements were done in ambient conditions at room temperature. The exposure time for each individual measurement was $20 \mathrm{~s}$.

\section{Data availability}

The authors declare that the main data supporting the findings of this study are available within the article and its Supplementary Information files. Extra data are available from the corresponding author upon request.

Received: 8 August 2018 Accepted: 31 August 2018

Published online: 27 September 2018

\section{References}

1. Ponomarenko, L. A. et al. Tunable metal-insulator transition in double-layer graphene heterostructures. Nat. Phys. 7, 958-961 (2011).

2. Hunt, B., Taniguchi, T., Moon, P., Koshino, M. \& Ashoori, R. C. Massive Dirac Fermions and hofstadter butterfly in a van der Waals heterostructure. Science 340, 1427-1431 (2013).

3. Evers, F. \& Mirlin, A. D. Anderson transitions. Rev. Mod. Phys. 80, 1355-1417 (2008).

4. Müller, M., Fritz, L. \& Sachdev, S. Quantum-critical relativistic magnetotransport in graphene. Phys. Rev. B 78, 115406 (2008).

5. Katsnelson, M. I. Zitterbewegung, chirality, and minimal conductivity in graphene. Eur. Phys. J. B 51, 157-160 (2006).

6. Tzalenchuk, A. et al. Towards a quantum resistance standard based on epitaxial graphene. Nat. Nanotechnol. 5, 186-189 (2010).

7. Janssen, T. et al. Operation of graphene quantum Hall resistance standard in a cryogen-free table-top system. 2D Mater. 2, 35015 (2015)

8. Wu, Y. et al. State-of-the-art graphene high-frequency electronics. Nano Lett. 12, 3062-3067 (2012).

9. Lin, Y. M. et al. $100-\mathrm{GHz}$ transistors from wafer-scale epitaxial graphene. Science 327, 662 (2010).

10. Kopylov, S., Tzalenchuk, A., Kubatkin, S. \& Fal'ko, V. I. Charge transfer between epitaxial graphene and silicon carbide. Appl. Phys. Lett. 97, 11-14 (2010).
11. Xue, J. et al. Scanning tunnelling microscopy and spectroscopy of ultraflat graphene on hexagonal boron nitride. Nat. Mater. 10, 282-285 (2011).

12. Mayorov, A. S. et al. How close can one approach the dirac point in graphene experimentally? Nano Lett. 12, 4629-4634 (2012).

13. Li, Q., Hwang, E. H. \& Das Sarma, S. Disorder-induced temperaturedependent transport in graphene: puddles, impurities, activation, and diffusion. Phys. Rev. B Condens. Matter Mater. Phys. 84, 115442 (2011).

14. Li, J. et al. Measurement of small molecular dopant F4TCNQ and C60F36 diffusion in organic bilayer architectures. ACS Appl. Mater. Interfaces 7, 28420-28428 (2015).

15. Jacobs, I. E. \& Moul, A. J. Controlling molecular doping in organic semiconductors. Adv. Mater. 29, 1703063 (2017).

16. Pingel, P. \& Neher, D. Comprehensive picture of p-type doping of P3HT with the molecular acceptor $\mathrm{F}_{4}$ TCNQ. Phys. Rev. B 87, 115209 (2013).

17. Li, J. et al. Quantitative measurements of the temperature-dependent microscopic and macroscopic dynamics of a molecular dopant in a conjugated polymer. Macromolecules 50, 5476-5489 (2017).

18. Lara-Avila, S. et al. Disordered Fermi liquid in epitaxial graphene from quantum transport measurements. Phys. Rev. Lett. 107, 166602 (2011).

19. Cho, S. \& Fuhrer, M. S. Charge transport and inhomogeneity near the minimum conductivity point in graphene. Phys. Rev. B Condens. Matter Mater. Phys. 77, 081402 (2008).

20. Tiwari, R. P. \& Stroud, D. Model for the magnetoresistance and Hall coefficient of inhomogeneous graphene. Phys. Rev. B Condens. Matter Mater. Phys. 79, 165408 (2009)

21. Ping, J. et al. Disorder-induced magnetoresistance in a two-dimensional electron system. Phys. Rev. Lett. 113, 047206 (2014).

22. Knap, M., Sau, J. D., Halperin, B. I. \& Demler, E. Transport in twodimensional disordered semimetals. Phys. Rev. Lett. 113, 186801 (2014).

23. Yang, M. et al. Puddle-induced resistance oscillations in the breakdown of the graphene quantum Hall effect. Phys. Rev. Lett. 117, 1-5 (2016).

24. Poumirol, J. M. et al. Electron-hole coexistence in disordered graphene probed by high-field magneto-transport. New J. Phys. 12, 083006 (2010).

25. Zhang, Y., Brar, V. W., Girit, C., Zettl, A. \& Crommie, M. F. Origin of spatial charge inhomogeneity in graphene. Nat. Phys. 5, 722-726 (2009).

26. Banszerus, L. et al. Ultrahigh-mobility graphene devices from chemical vapor deposition on reusable copper. Sci. Adv. 1, e1500222-e1500222 (2015).

27. Chen, W., Chen, S., Dong, C. Q., Xing, Y. G. \& Wee, A. T. S. Surface transfer p-type doping of epitaxial graphene. J. Am. Chem. Soc. 129, 10418-10422 (2007).

28. Coletti, C. et al. Charge neutrality and band-gap tuning of epitaxial graphene on SiC by molecular doping. Phys. Rev. B Condens. Matter Mater. Phys. 81, 235401 (2010).

29. Li, Q., Hwang, E. H., Rossi, E. \& Das Sarma, S. Theory of 2D transport in graphene for correlated disorder. Phys. Rev. Lett. 107, 156601 (2011).

30. Yan, J. \& Fuhrer, M. S. Correlated charged impurity scattering in graphene. Phys. Rev. Lett. 107, 206601 (2011).

31. Yang, S., Jiang, Y., Li, S. \& Liu, W. Many-body dispersion effects on the binding of TCNQ and F4-TCNQ with graphene. Carbon N. Y. 111, 513-518 (2017).

32. Mao, J. et al. Tunability of supramolecular kagome lattices of magnetic phthalocyanines using graphene-based moiré patterns as templates. J. Am. Chem. Soc. 131, 14136-14137 (2009).

33. Cho, J. et al. Structural and electronic decoupling of C 60 from epitaxial graphene on sic. Nano Lett. 12, 3018-3024 (2012).

34. Wang, Q. H. \& Hersam, M. C. Room-temperature molecular-resolution characterization of self-assembled organic monolayers on epitaxial graphene. Nat. Chem. 1, 206-211 (2009).

35. Tsai, H.-Z. et al. Molecular self-assembly in a poorly screened environment: $\mathrm{F}$ 4 TCNQ on graphene/BN. ACS Nano 9, 12168-12173 (2015).

36. Virojanadara, C. et al. Homogeneous large-area graphene layer growth on $6 \mathrm{H}-$ SiC(0001). Phys. Rev. B 78, 245403 (2008).

37. Yager, T. et al. Express optical analysis of epitaxial graphene on SiC: impact of morphology on quantum transport. Nano Lett. 13, 4217-4223 (2013).

38. Yager, T. et al. Low contact resistance in epitaxial graphene devices for quantum metrology. AIP Adv. 5, 87134 (2015).

\section{Acknowledgements}

We thank Dmitry Golubev, Alexander Tzalenchuk, and Tord Claeson for useful discussion and critical reading of the manuscript. This work was jointly supported by the Swedish Foundation for Strategic Research (SSF) (No. IS14-0053, GMT14-0077, and RMA15-0024), Knut and Alice Wallenberg Foundation, Chalmers Area of Advance NANO, the Swedish Research Council (VR) 2015-03758 and 2016-048287, the Swedish-Korean Basic Research Cooperative Program of the NRF (No. NRF-2017R1A2A1A18070721), and European Union's Horizon 2020 research and innovation program (grant agreement No. 766714/HiTIMe). We thank Cornell 
High Energy Synchrotron Source (CHESS) for providing time for GIWAXs measurements. CHESS is supported by the NSF \& NIH/NIGMS via NSF award DMR-1332208.

\section{Author contributions}

H.H. and S.L.-A. fabricated samples, performed transport measurements, and analyzed data. K.H.K., A.D., D.M., F.L., Y.W.P. and T.B. performed transport experiments. P.M. performed and analyzed SIMS measurements. K.M.-P., L.Y. and C.M. analyzed GIWAXS measurements. H.H. and S.L.A. wrote the manuscript, with contributions from all of the authors. S.K. and S.L.A. conceived and designed the experiment.

\section{Additional information}

Supplementary Information accompanies this paper at https://doi.org/10.1038/s41467018-06352-5.

Competing interests: The authors declare no competing interests.

Reprints and permission information is available online at http://npg.nature.com/ reprintsandpermissions/
Publisher's note: Springer Nature remains neutral with regard to jurisdictional claims in published maps and institutional affiliations.

\section{(c) (1)}

Open Access This article is licensed under a Creative Commons Attribution 4.0 International License, which permits use, sharing, adaptation, distribution and reproduction in any medium or format, as long as you give appropriate credit to the original author(s) and the source, provide a link to the Creative Commons license, and indicate if changes were made. The images or other third party material in this article are included in the article's Creative Commons license, unless indicated otherwise in a credit line to the material. If material is not included in the article's Creative Commons license and your intended use is not permitted by statutory regulation or exceeds the permitted use, you will need to obtain permission directly from the copyright holder. To view a copy of this license, visit http://creativecommons.org/ licenses/by/4.0/.

(C) The Author(s) 2018 